\title{
Oblique Lumbar Interbody Fusion at the L5-S1 Segment via an Approach Between the Psoas Muscle and the Great Vessels: Technique Note and Case Presentations.
}

\section{Xigong Li}

Zhejiang University School of Medicine First Affiliated Hospital

\section{Weiyi Diao}

Zhejiang University School of Medicine First Affiliated Hospital

Yuzhu Zhang ( $\sim$ zhangyuzhu1963@163.com )

Zhejiang University School of Medicine First Affiliated Hospital Junsong Wu

Zhejiang University School of Medicine First Affiliated Hospital Chunyang Xing

Zhejiang University School of Medicine First Affiliated Hospital Junhua Du

Zhejiang University School of Medicine First Affiliated Hospital

\section{Bin Chen}

Zhejiang University School of Medicine First Affiliated Hospital

\section{Sanzhong Xu}

Zhejiang University School of Medicine First Affiliated Hospital

Research article

Keywords: OLIF, L5-S1, Psoas muscle, The great vessels, Spine surgery

Posted Date: December 15th, 2020

DOl: https://doi.org/10.21203/rs.3.rs-125260/v1

License: (c) (1) This work is licensed under a Creative Commons Attribution 4.0 International License. Read Full License 


\section{Abstract}

Study Design

Technique note.

Objectives

To describe our modified oblique lumbar interbody fusion (OLIF) technique in the reconstruction of the L5-S1 segment.

Summary of Background Data

Recently, OLIF has been generally recognized as an effective procedure in the treatment of various spinal pathologies at L2-L5 segments. However, the usage of OLIF at the L5-S1 segment doesn't have gained widespread acceptance in spine community. Some authors still concern about the feasibility of OLIF used in lumbosacral fusion

Methods

Ten consecutive patients underwent L5-S1 interbody fusion using the OLIF technique in our institution. The L5-S1 disc space is approached via one retroperitoneal oblique corridor between the psoas muscle and the great vessels. The discectomy and endplate preparation are performed through a surgical window developed on the anterolateral side of L5-S1 disc. A secondary cage insertion technique is used for safe placement of interbody fusion cages.

Results

Of the 10 patients, 6 were males and 4 were females, with an average age of $55.4 \pm 6.8$ years. There were 8 single-level and 2 two-level procedures, including 2 at L4-L5 and 10 at L5-S1. Preoperative axial MR images confirmed 1 patient with type I LCIV (left common iliac vein), 6 with type II LCIV and 3 with type III LCIV. The average blood loss was $133.4 \pm 88.5 \mathrm{ml}$, and the average operative times were $153.6 \pm 38.3$ minutes. Postoperative radiographs examination confirmed all patients obtained a better reconstruction at the lumbosacral junction. Two patients with type III LCIV sustained iliolumbar vein laceration during the exposure, and no other perioperative complications were encountered.

Conclusion

Our novel OLIF L5-S1 technique is a more feasible procedure of lumbosacral fusion, which shared the common surgical plane with OLIF L2-5, allowing for L2 to S1 reproducible multi-levels interbody fusions via a retroperitoneal oblique corridor between the psoas muscle and the great vessels. Detailed preoperative plan and meticulous intraoperative manipulation are prerequisite for the success of OLIF L5S1 procedure. 


\section{Background}

Oblique lumbar interbody fusion (OLIF) is an innovative surgical technique for reconstruction of the lumbar spine [1-4]. As an alternative technique to lateral lumbar interbody fusion (LLIF), the OLIF procedure has several theoretical advantages over LLIF, such as avoiding psoas muscle disruption and lumbosacral plexus injury, consistent access to the lower lumbar levels without the need for neuromonitoring $[3,4]$. Recently, OLIF has been generally recognized as an effective procedure in the treatment of various spinal pathologies at L2-L5 segments, including degenerative disc disease, lumbar spondylosis, spondylolisthesis, lumbar stenosis and scoliosis [2, 5-7].

With the advancement in surgical techniques and instruments, several spine surgeons attempt to perform L5-S1 interbody fusion with the OLIF technique (termed as OLIF L5-S1) [2, 6-12]. The usage of OLIF at the lumbosacral junction can provide larger surface area for solid fusion, reestablish anterior column support, and simultaneously achieve indirect decompression and correction of sagittal alignment. However, the anatomic characteristics of the retroperitoneal oblique corridor to the L5-S1 disc pose technical challenges to spine surgeons, especially the complexity of neurovascular structures and disturbance of the iliac crest $[2,9,11,12-14]$. Despite few literatures related to OLIF L5-S1 are available in recent years, OLIF L5-S1 doesn't have gained widespread acceptance in spine community as OLIF L2-5. Some authors still concern about the feasibility of OLIF L5-S1 used in lumbosacral fusion $[2,13,14]$.

In the present study, we describe the procedure of our OLIF L5-S1 technique with case illustrations. Our novel technique can allow for reproducible arthrodesis of the L5-S1 segment via one retroperitoneal oblique corridor between the psoas muscle and the great vessels.

\section{Methods}

\section{Indication and baseline evaluation}

The OLIF L5-S1 technique is indicated for patients with various spinal pathologies of L5-S1 involving degenerative disc disease, lumbar spondylosis, low-grade spondylolisthesis, and scoliosis. All patients were diagnosed on radiograph, computed tomography (CT), and magnetic resonance imaging (MRI). Preoperatively, the length of the selected cage is initially measured on axial CT image at the level of L5S1. The morphology of the left common iliac vein (LCIV) at the L5-S1 disc was assessed on axial MR images, and categorized into three types according to the classification of Chung et al [15]: type I no requirement for mobilizing LCIV; type II easily mobilizing LCIV; type III difficultly mobilizing LCIV

\section{Surgical technique}

The patient is positioned in the right or left lateral decubitus on a radiolucent table, with the greater trochanter and shoulder fixation (Fig 1a, b). A left-sided approach is generally preferred to reduce the risk of the great vein injury, in condition of multi-level spine reconstruction involving L5-S1 junction. On the 
contrary, a right-sided approach may be indicated in patients with a scoliotic spine convex, and previous abdominal surgery on the left side.

Our technique for OLIF L5-S1 approach is described detailedly as below (video). Using fluoroscopy, the location of anterior superior iliac spine (ASIA) and L5-S1 disc are marked on the skin. Approximately $2 \mathrm{~cm}$ anterior to ASIA, an about $4 \mathrm{~cm}$ oblique anterolateral incision is made centering towards the landmark midpoint of the L5-S1 disc (Fig 1b). After performing a skin incision, the external oblique, internal oblique, and transversus abdominis muscles are split successively in the line of their fibers (Fig 1c). Blunt split is used to open transversal fascia and subsequently access the retroperitoneal space. Then the retroperitoneal fat along with the peritoneum and ureter can be swept anteriorly using the tonsil sponges. The retroperitoneal dissection continues over the anterior border of the psoas muscle until the iliac vessels can be identified. The left iliac artery is gently mobilized anteromedially to expose the entry to the L5-S1 disc guided by LCIV. In fact, the surgical plane of L5-S1 utilized in our procedures is requiring passage between the psoas muscle and the LCIV. It is a really extensile oblique trajectory that allows psoas-preserving access to L2-S1 disc space. In contrast to OLIF L2-5 technique, only a limited amount of exposure is needed at the intervals, in order to avoid intraoperative venous laceration and psoas retraction-related injury. A suction apparatus is generally used to bluntly dissect the loose fatty connective tissue at this region. Eventually, about a $1.8 \mathrm{~cm}$ surgical window can be carefully developed on the anterolateral side of L5-S1 disc (Fig 1d), which provides adequate working zone for discectomy and cage insertion. We emphasize both direct visualization and meticulous manipulation are prerequisite for safe operation in this procedure. Therefore, a smaller arthroscopy (STORZ 28208BA, Germany) is preferred to help the surgeons discern the neurovascular structures crossing the surgical window. The iliolumbar vein can be encountered on the entry point of L5-S1 disc, but can be preserved unless the surgical window is obstructed by the iliolumbar vein. In this situation, the iliolumbar vein should be controlled with bipolar cautery or clipped prior to discectomy.

Once the L5-S1 level is confirmed by fluoroscopy (Fig 1e), we prefer a handled retractor and several Kwires to maintain the adequate exposure, rather than using the expansion retractors. A handled retractor is placed laterally to the iliac vessels with gentle retraction. The Kirschner wires are respectively inserted into the L5 and S1 vertebral bodies under visualization of arthroscopy, which makes it possible to minimize retraction of the psoas major muscle and block the iliolumbar vein outside the surgical window. The Kirschner wires should be inserted close to the end-plate with minimal risk of neurovascular injury.

The discectomy and endplate preparation are performed through the developed surgical window. Different sizes of the trials are sequentially used to distract the disc space and release the contralateral annulus (Fig $1 \mathrm{f}-\mathrm{h}$ ). However, caution is needed to not perforate the contralateral annulus, as the right iliac veins get very close to the contralateral side of the L5-S1 disc. Based on preoperative templating and intraoperative trial sizing, a proper sized cage will be placed after filling it with bone graft and bone marrow aspirated from the iliac crest. At the level of L5-S1 disc, the high-riding iliac crest disturbs the orthogonal maneuver of the inserted instrument as usual. The secondary cage insertion technique is designed to avoid the obstruction of iliac crest at L5-S1(Fig 2a-f). The final implant begins to obliquely 
enter the disc space until approximately $75 \%$ of the cage is inserted (Fig 2a-C). Then instead of the conventional instrument, a shorter implant inserter is preferred to tap the anteromedial corner of the cage, which can be turned orthogonally and further inserted simultaneously (Fig $2 \mathrm{~d}-\mathrm{f}$ ). During the insertion of the cage, it is necessary to have a strict radiological anteroposterior and lateral radioscopic control until the satisfactory cage position is obtained intraoperatively.

The Kirschner wires are removed and the surgical field was irrigated with normal saline. A drain was inserted through the lateral surgically exposed area, and closure is performed in a layered fashion with an interrupted absorbable suture. In our series, all the patients underwent open posterolateral pedicular fixation at the same day. No intraoperative monitoring was used in all procedures, and no access surgeon took part in the retroperitoneal exposure.

\section{Case presentation}

A 51-year-old man presented with a 10-year history of lower back pain, bilateral buttock and thigh pain. On physical examination, the lumbosacral step-off sign was positive, but the neurological assessment was intact. His standing lateral radiograph showed that spondylolytic spondylolisthesis was noted at the level of L4-L5 and L5-S1, respectively (Fig 3a, b). Dynamic flexion-extension lateral radiographs of the lumbar spine demonstrate segmental instability within the two olisthetic levels (Fig 3c, d). The preoperative sagittal MR image showed that marked disk degeneration at the L4-L5 and L5-S1 segments (Fig 3e). The axial MR image showed that perivascular adipose tissues were still observed beneath the LCIV at the level of central L5-S1 disk space (Fig 3f). The patient's symptom aggravated continuously after 6 months of conservative treatment. Therefore, OLIF and posterior pedicle screws fixation were performed from the L4-L5 level to the L5-S1 level to reconstruct the stability across the lumbosacral junction. There were no perioperative complications, and postoperative radiographs showed that a satisfactory L4-S1 reconstruction with good cage position (Fig $3 g, h$ ).

\section{Results}

A total of ten patients underwent OLIF L5-S1 via a retroperitoneal oblique corridor between the psoas muscle and the great vessels (Table 1). Of the 10 patients, 6 were males and 4 were females, with an average age of $55.4 \pm 6.8$ years. OLIF L5-S1 was performed in 4 patients with isthmic spondylolisthesis, 4 with degenerative disc disease, and 2 with degenerative spondylolisthesis. There were 8 single-level and 2 two-level procedures, including 2 at L4-L5 and 10 at L5-S1. Preoperative axial MR images confirmed 1 patient with type I LCIV, 6 with type II LCIV and 3 with type III LCIV. The average blood loss was $133.4 \pm 88.5$ $\mathrm{ml}$, and the average operative times were $153.6 \pm 38.3$ minutes. Postoperative radiographs examination confirmed all patients obtained a better reconstruction at the lumbosacral junction. Two patients with type III LCIV sustained iliolumbar vein laceration during the exposure, and there were no other perioperative complications observed in our series.

\section{Discussion}


Recently, a few of clinical studies have demonstrated the technical advantages and favorable outcomes of OLIF therapy for lumbar degenerative diseases, mainly involving the L2-5 segments [2, 5-7]. Bearing these benefits in mind, there has been cautious endeavor for the usage of OLIF at the lumbosacral junction. However, the current technique strategy for performing OLIF at L5-S1 remains controversial in the spine surgery community $[2,13,14]$.

Base on their previous experience with anterior lumbar interbody fusion (ALIF), several authors suggest the OLIF L5-S1 procedure should be performed through the central part of the L5-S1 disc, below the bifurcation of the iliac vessels $[6-8,11,13]$. In essence, this technique is deemed to be a minimally invasive retroperitoneal ALIF in the lateral position [16], and the surgical plane utilized in the procedure is not essentially the same accessible corridor as in OLIF L2-5. Therefore, it is necessary to change the position of patients for facilitating better manipulation while multiple-level fusion procedure including the L5-S1 segment, is required to be done in one-stage operation. And the integrity of anterior discoligamentous complex (anterior longitudinal ligament and annulus) is sacrificed in this procedure, which may weaken the effectiveness of OLIF in indirect neural decompression. As we know, the superior hypogastric plexus lies directly over the L5-S1 disc space below the bifurcation of the iliac vessels. The central access route to the L5-S1 disc space between the iliac vessels may increase the risk of injury to the superior hypogastric plexus and the resultant retrograde ejaculation [16]. Additionally, in some cases with aberrant vascular structures, the low iliocava junction or the medially located left common iliac vein make the approach very difficult and may result in life-threatening vascular injury $[17,18]$.

In order to avoid these disadvantages, Silvestre et al attempts to access the L5-S1 disc through the oblique corridor between the iliac vessel and the psoas muscle. However, one major concern for damage to the iliac vessel and its tributaries such as the iliolumbar vein, compels the surgeons to abandon the usage of OLIF at the L5-S1 level [2]. Zairi et al suggests ligating the iliolumbar vein should be prerequisite for successful access to the L5-S1 disc, with aim of reducing the incidental injury of these vulnerable structures [9]. However, it generally requires excessive dissection and manipulation with more soft tissue interference around the oblique corridor, potentially increase postoperative thigh pain and the chance of neurovascular complications. And the searching process of the iliolumbar vein may be complicated by the variability of the vascular anatomy encountered during the exposure.

In our experience, the distinctness of the OLIF L5-S1 procedure requires that surgeons should be well familiarized with the lumbosacral anatomy. The true accessible corridor in the procedure is located at the anatomic space between the psoas muscle and the LCIV. Kai et al in a cadaveric study found the mean distance from the LCIV to the psoas muscle was $12.00 \mathrm{~mm}$ at the L5-S1 disc space [14]. Mild psoas retraction can efficiently increase the width of the accessible corridor to $18 \mathrm{~mm}$, which is adequate for introduction of cages into the disc space. In addition, the limited space available for access to the L5-S1 disc may restrict the use of the expansion retractors. Alternatively, the Kirschner wire is preferred in our procedure to maintain enough exposure using its elastic retraction. As our technique improved, we require lesser dissection and retraction to achieve adequate working zone for OLIF L5-S1, suggesting an easier method than previously described. We emphasize the importance of careful dissection and direct 
visualization in safe exposure. Any dissection should be carried out under direct visualization of the anatomic landmarks. Meanwhile, a smaller arthroscopy is utilized in our procedure to gain more detailed view of the important anatomy crossing the surgical window, especially the location of the iliolumbar vein. Generally, the iliolumbar vein can be protected from being incidentally damaged in our procedure, obviating the need for vascular ligation and resultant extensive exposure.

The implant insertion in the presence of the iliac crest is another technical challenge of the OLIF L5-S1 procedure $[2,9,11]$. Obstruction of the iliac crest at the L5-S1 level poses some difficulty for the orthogonal maneuver of the inserted instrument, which may lead to postoperative misplacement of interbody fusion cages. In our series, the secondary insertion technique is adopted to turn the implants orthogonal to the true lateral direction until the satisfactory cage position is obtained under fluoroscopy monitoring. Our surgical strategy for cage insertion will make sense to apply the same OLIF device system for L2-S1 consecutive reconstruction through the same surgical plane, which is a more universal procedure in practice.

Last but most importantly, we believe that a careful preoperative analysis of the vascular anatomy is essential when performing OLIF at the L5-S1 level. Chung et al in a MRI anatomic study found the morphological characteristics of LCIV is markedly associated with the risk of mobilization at the L5-S1 level [15]. Both the position of LCIV and presence of perivascular adipose tissue should be taken into consideration when approaching the L5-S1 disc between the iliac vessels. This classification system is not completely appropriate for the assessment of LCIV in our approach. However, in our experience, the absence of the fats around LCIV leave the adventitial layer tightly adhesive to the surrounding connective tissue, which indeed poses more difficulty for blunt dissection and retraction. During the exposure, the anatomic features of type III LCIV will bring the higher risk of venous laceration to the accessible surgeons. We therefore attach importance to preoperative evaluation of perivascular adipose tissue on axial MR image, and do not recommended the OLIF technique for one single L5-S1 fusion in condition of type III LCIV.

In conclusion, our novel OLIF L5-S1 technique is a more feasible procedure of lumbosacral fusion, which shared the common surgical plane with OLIF L2-5, allowing for L2 to S1 reproducible multi-levels interbody fusions via a retroperitoneal oblique corridor between the psoas muscle and the great vessels. Detailed preoperative plan and meticulous intraoperative manipulation are prerequisite for the success of OLIF L5-S1 procedure.

\section{Abbreviations}

OLIF: oblique lumbar interbody fusion

LCIV: left common iliac vein

LLIF: lateral lumbar interbody fusion 
CT: computed tomography

MRI: magnetic resonance imaging

AISA: anterior superior iliac spine

\section{Declarations}

Ethics approval and consent to participate:

This study has been approved by the Ethics Committee of The First Affiliated Hospital of Zhejiang University. Written consent to participate in this study was obtained from all the participants of this study.

Consent for publication:

Not applicable

Availability of data and materials:

Not applicable

Competing interests:

Not applicable

Funding:

Not applicable

Authors' contributions:

XL: Research conduction and manuscript drafting; WD: Manuscript revision; ZZ: Research designing; JW: Data collection; CX: Data analysis; JD: Surgical strategy guidance; BC: Manuscript review; SX: Ethical support.

\section{Acknowledgements:}

Not applicable

Authors' information:

Not applicable

All Authors read and approved the manuscript.

\section{References}


1. Mobbs RJ, Phan K, Malham G, et al. Lumbar interbody fusion: techniques, indications and comparison of interbody fusion options including PLIF, TLIF, MI-TLIF, OLIF/ATP, LLIF and ALIF. $J$ Spine Surg 2015, 1(1):2.

2. Silvestre, Clément, Mac-Thiong JM, et al. Complications and morbidities of mini-open anterior retroperitoneal lumbar interbody fusion: oblique lumbar interbody fusion in 179 patients. Asian Spine $J$ 2012, 6(2).

3. Phan K, Maharaj M, Assem Y, et al. Review of early clinical results and complications associated with oblique lumbar interbody fusion (OLIF). J Clinic Neurosci 2016, S0967586816300236.

4. Hah R, Kang HP. Lateral and oblique lumbar interbody fusion-current concepts and a review of recent literature. Curr Rev Musculoskelet Med 2019 (Suppl 3).

5. Fujibayashi S, Hynes RA, Otsuki B, et al. Effect of indirect neural decompression through oblique lateral interbody fusion for degenerative lumbar disease. Spine 2015, 40(3): E175-82.

6. Molloy S, Butler JS, Benton A, et al. A new extensile anterolateral retroperitoneal approach for lumbar interbody fusion from L1 to S1: a prospective series with clinical outcomes. Spine J 2016 : S1529943016005027.

7. Kim KT, Jo DJ, Lee SH, et al. Oblique retroperitoneal approach for lumbar interbody fusion from L1 to S1 in adult spinal deformity. Neurosurg Rev 2017.

8. Kim JS, Sharma SB. How I do it? Oblique lumbar interbody fusion at L5S1(OLIF51). Acta Neurochi 2019.

9. Zairi F, Sunna TP, Westwick HJ, et al. Mini-open oblique lumbar interbody fusion (OLIF) approach for multi-level discectomy and fusion involving L5-S1: Preliminary experience. Orthop Traumatol Surg Res 2017, 103(2):295-9.

10. Pham MH, Jakoi AM, Hsieh PC. Minimally invasive L5-S1 oblique lumbar interbody fusion with anterior plate. Neurosurgical Focus 2016, 41(VideoSuppl1):1-1.

11. Kanno K, Ohtori S, Orita S, et al. Miniopen oblique lateral L5-S1 interbody fusion: a report of 2 cases. Case Rep Orthop 2014, 2014:603531.

12. Chung NS, Jeon $\mathrm{CH}$, Lee HD. Use of an alternative surgical corridor in oblique lateral interbody fusion at the L5-S1 segment. Clin Spine Surg 2017: 1.

13. Davis TT, Hynes RA, Fung DA, et al. Retroperitoneal oblique corridor to the L2-S1 intervertebral discs in the lateral position: an anatomic study. J Neurosurg Spine 2014, 21:785-93.

14. Kai W, Can Z, Hao W, et al. The anatomic characteristics of the retroperitoneal oblique corridor to the L1-S1 intervertebral disc spaces. Spine 2018.

15. Chung NS, Jeon CH, Lee HD, et al. Preoperative evaluation of left common iliac vein in oblique lateral interbody fusion at L5-S1. Eur Spine J 2017, 26:2797-803.

16. Eid S, Iwanaga J, Chapman JR, et al. Superior hypogastric plexus and its surgical implications during spine surgery: a review. World Neurosur 2018, 120:163-7. 
17. Tribus CB, Belanger T. The vascular anatomy anterior to the L5-S1 disk space. Spine 2001, 26(11):1205-8.

18. Fantini G. Major vascular injury during anterior lumbar spinal surgery: incidence, risk factors, and management. Spine 2007, 32:2751-8.

\section{Tables}

Table 1. Case Series of the OLIF L5-S1 procedure

\begin{tabular}{|c|c|c|c|c|c|c|c|c|}
\hline No. & Gender & $\begin{array}{l}\text { Age } \\
\text { (yrs) }\end{array}$ & Level & Diagnose & $\begin{array}{l}\text { Operation } \\
\text { time } \\
\text { (min) }\end{array}$ & $\begin{array}{l}\text { Blood } \\
\text { loss } \\
(\mathrm{ml})\end{array}$ & $\begin{array}{l}\text { Type } \\
\text { of } \\
\text { LICV }\end{array}$ & Complications \\
\hline 1 & $M$ & 57 & $\begin{array}{l}\text { L4- } \\
\text { S1 }\end{array}$ & $\begin{array}{l}\text { isthmic } \\
\text { spondylolisthesis }\end{array}$ & 196 & 110 & Type & None \\
\hline 2 & $\mathrm{~F}$ & 53 & $\begin{array}{l}\text { L5- } \\
\text { S1 }\end{array}$ & $\begin{array}{l}\text { degenerative disc } \\
\text { disease }\end{array}$ & 123 & 80 & $\begin{array}{l}\text { Type } \\
\|\end{array}$ & None \\
\hline 3 & $M$ & 44 & $\begin{array}{l}\text { L5- } \\
\text { S1 }\end{array}$ & $\begin{array}{l}\text { isthmic } \\
\text { spondylolisthesis }\end{array}$ & 208 & 300 & $\begin{array}{l}\text { Type } \\
\text { III }\end{array}$ & $\begin{array}{l}\text { iliolumbar } \\
\text { vein injury }\end{array}$ \\
\hline 4 & $M$ & 56 & $\begin{array}{l}\text { L5- } \\
\text { S1 }\end{array}$ & $\begin{array}{l}\text { degenerative disc } \\
\text { disease }\end{array}$ & 110 & 100 & $\begin{array}{l}\text { Type } \\
\text { II }\end{array}$ & None \\
\hline 5 & $\mathrm{~F}$ & 70 & $\begin{array}{l}\text { L5- } \\
\text { S1 }\end{array}$ & $\begin{array}{l}\text { degenerative disc } \\
\text { disease }\end{array}$ & 123 & 80 & $\begin{array}{l}\text { Type } \\
\text { ॥ }\end{array}$ & None \\
\hline 6 & $M$ & 57 & $\begin{array}{l}\text { L5- } \\
\text { S1 }\end{array}$ & $\begin{array}{l}\text { isthmic } \\
\text { spondylolisthesis }\end{array}$ & 145 & 85 & $\begin{array}{l}\text { Type } \\
\text { II }\end{array}$ & None \\
\hline 7 & $\mathrm{~F}$ & 58 & $\begin{array}{l}\text { L5- } \\
\text { S1 }\end{array}$ & $\begin{array}{l}\text { degenerative disc } \\
\text { disease }\end{array}$ & 148 & 100 & $\begin{array}{l}\text { Type } \\
\text { III }\end{array}$ & None \\
\hline 8 & $M$ & 50 & $\begin{array}{l}\text { L5- } \\
\text { S1 }\end{array}$ & $\begin{array}{l}\text { degenerative disc } \\
\text { disease }\end{array}$ & 132 & 300 & $\begin{array}{l}\text { Type } \\
\text { III }\end{array}$ & $\begin{array}{l}\text { iliolumbar } \\
\text { vein injury }\end{array}$ \\
\hline 9 & M & 58 & $\begin{array}{l}\text { L5- } \\
\text { S1 }\end{array}$ & $\begin{array}{l}\text { degenerative disc } \\
\text { disease }\end{array}$ & 136 & 80 & $\begin{array}{l}\text { Type } \\
\text { II }\end{array}$ & None \\
\hline 10 & $\mathrm{~F}$ & 51 & $\begin{array}{l}\text { L4- } \\
\text { S1 }\end{array}$ & $\begin{array}{l}\text { isthmic } \\
\text { spondylolisthesis }\end{array}$ & 215 & 100 & $\begin{array}{l}\text { Type } \\
\text { ॥ }\end{array}$ & None \\
\hline
\end{tabular}

Yrs indicates years; Min, minutes; LICV, left iliac common vein; $M$, male; $F$, female.

\section{Figures}




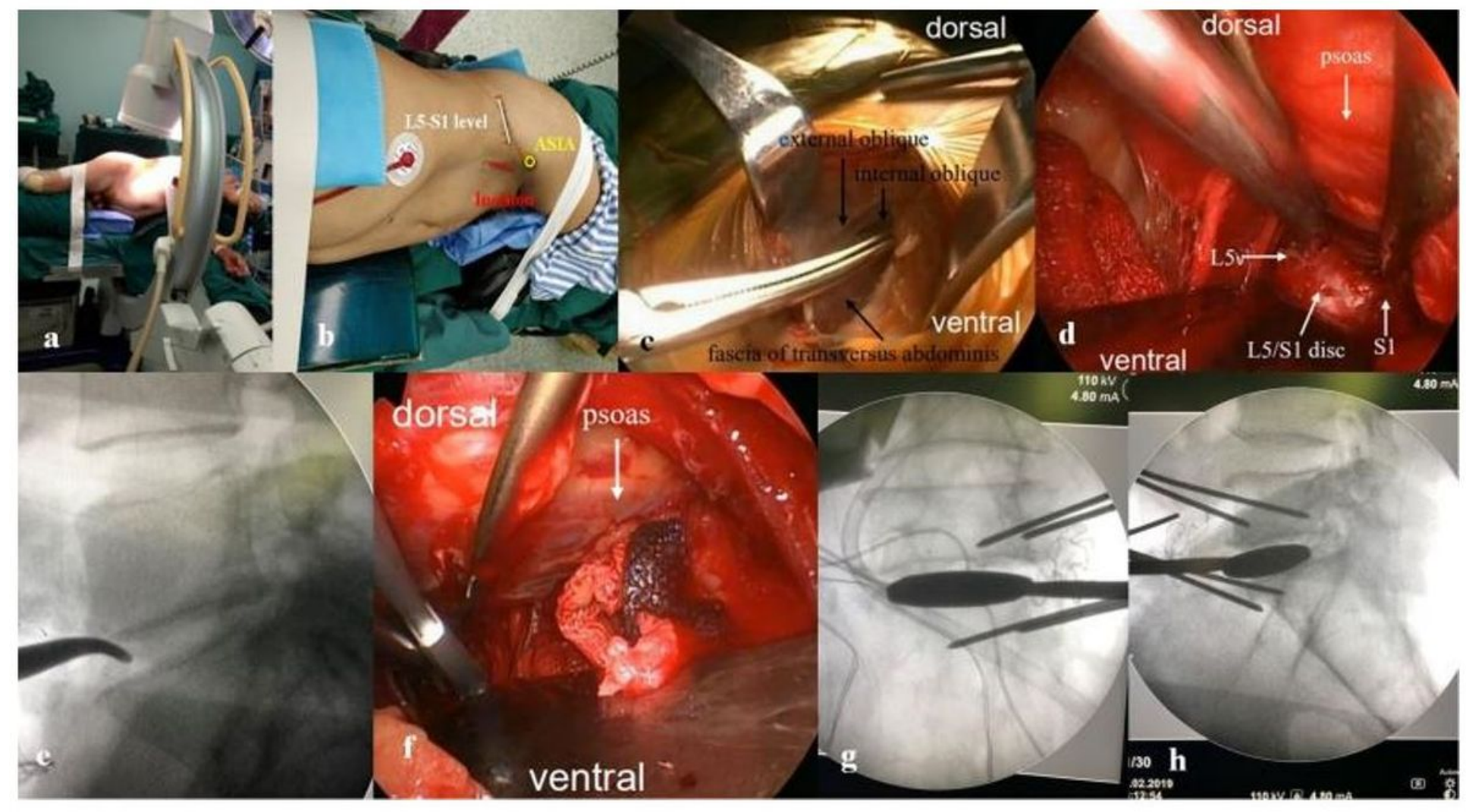

\section{Figure 1}

The lateral decubitus position of the patient and and skin marking for incision (a-b). Blunt dissection splitting fibers of external oblique, internal oblique, and transversus abdominis muscles (c). The L5-S1 disc space is exposed using handheld retractors and K-wire pins (d). The L5-S1 disc space is confirmed intraoperatively by fluoroscopy (e). The different sizes of trials are sequentially used to distract the disc space and release the contralateral annulus (f-h). 


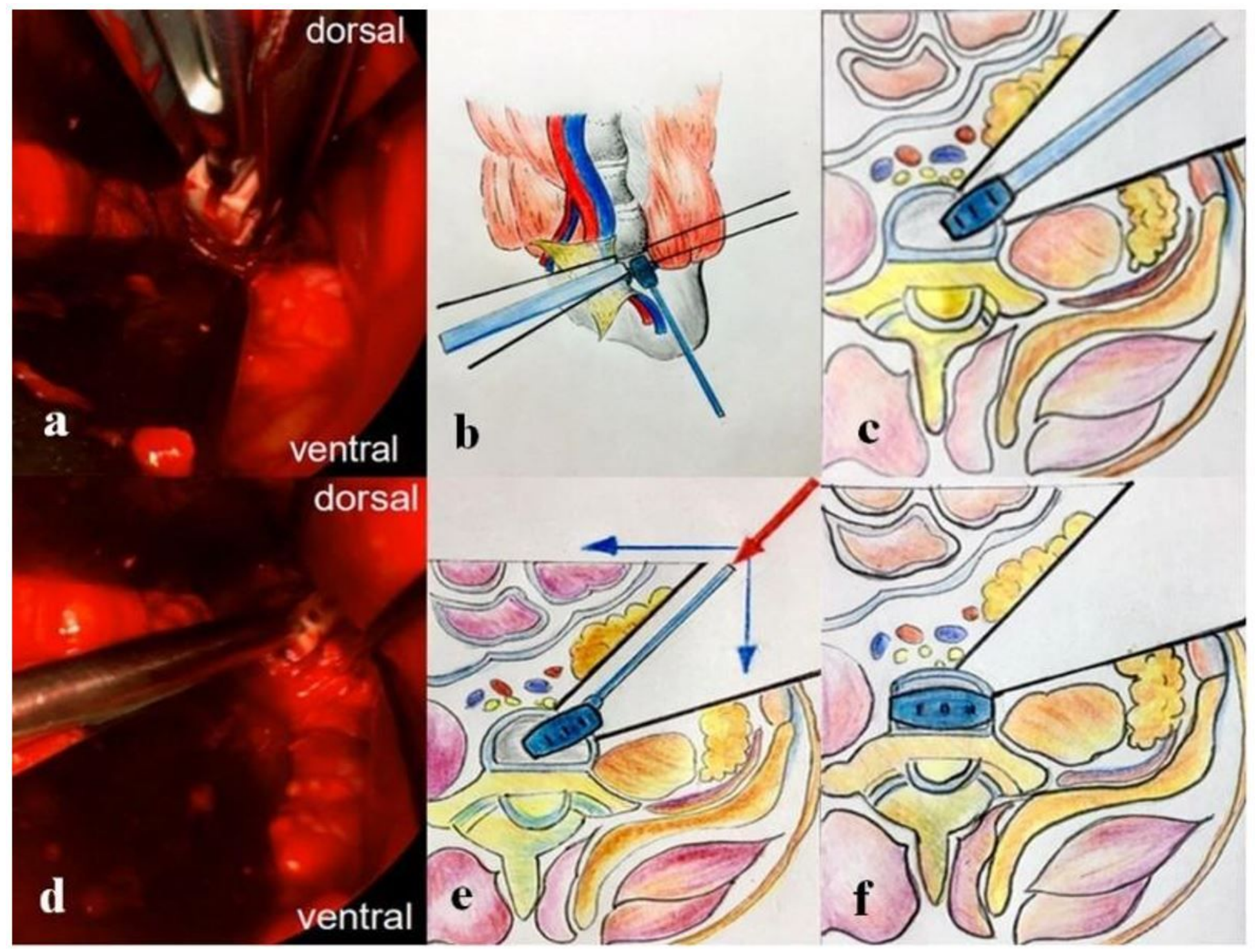

Figure 2

Intraoperative clinical pictures and schematic diagrams of cage insertion technique at the L5-S1 segment. (a-c) the first step of cage insertion. (d-f) the second step of cage insertion.

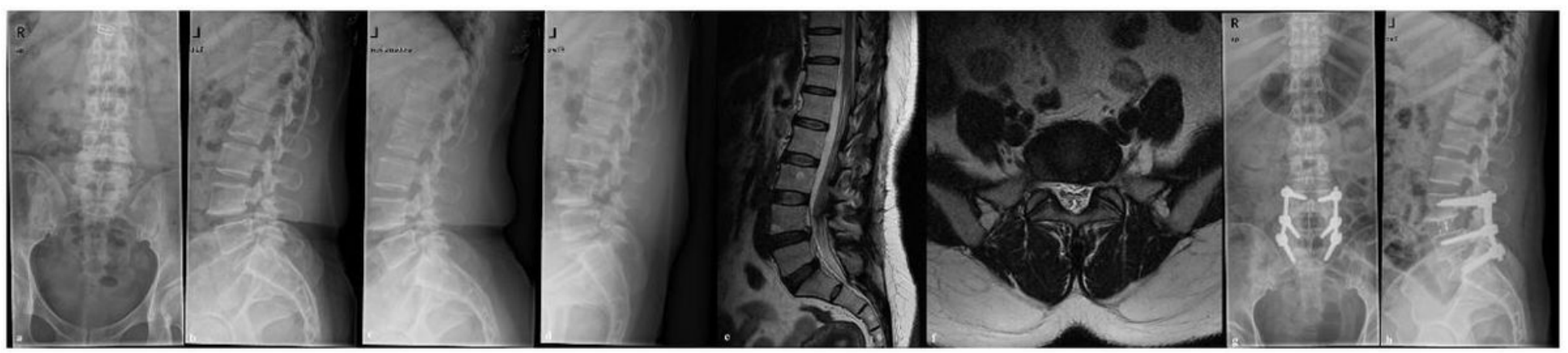

Figure 3

A 51-year-old man underwent OLIF from the L4-5 level to the L5-S1 level. Preoperative anteroposterior and lateral radiographs showed that spondylolytic spondylolisthesis at the level of L4-L5 and L5-S1 (a, 
b). Dynamic flexion-extension lateral radiographs of the lumbar spine demonstrate segmental instability of the two olisthetic levels (c, d). Preoperative sagittal MR image showed that disk degeneration at the L4L5 and L5-S1 segments (e). Preoperative axial MR image showed that perivascular adipose tissues were noted beneath the LCIV. The axial MR image showed that perivascular adipose tissues were observed beneath the LCIV at the level of central L5-S1 disk space (f). Postoperative anteroposterior and lateral radiographs showed that a satisfactory L4-S1 reconstruction $(g, h)$.

\section{Supplementary Files}

This is a list of supplementary files associated with this preprint. Click to download.

- Vedio.mp4 